IdeAs

Idées d'Amériques

18 | 2021

Frontières dans les Amériques - Intégration, sécurité

et migrations

\title{
Costa Rica aux portes du bicentenaire : portrait d'une société, portrait d'une crise
}

\section{Eduardo Madrigal}

\section{(2) OpenEdition \\ Journals}

Édition électronique

URL : https://journals.openedition.org/ideas/11478

DOI : $10.4000 /$ ideas. 11478

ISSN : 1950-5701

Éditeur

Institut des Amériques

Référence électronique

Eduardo Madrigal, « Costa Rica aux portes du bicentenaire : portrait d'une société, portrait d'une crise », IdeAs [En ligne], 18 | 2021, mis en ligne le 01 octobre 2021, consulté le 21 octobre 2021. URL : http://journals.openedition.org/ideas/11478; DOI : https://doi.org/10.4000/ideas.11478

Ce document a été généré automatiquement le 21 octobre 2021.

\section{cc)}

IdeAs - Idées d'Amériques est mis à disposition selon les termes de la licence Creative Commons Attribution - Pas d'Utilisation Commerciale - Pas de Modification 4.0 International. 


\title{
Costa Rica aux portes du bicentenaire : portrait d'une société, portrait d'une crise
}

\author{
Eduardo Madrigal
}

1 Chaque année, le 15 septembre, le Costa Rica célèbre son indépendance (survenue en 1821) sous l'aile d'un discours politique officiel qui en propage une image fantasmée : le moment de la naissance d'une nation certes moderne, mais aussi idyllique et romantique, bref, le point de départ de l'État-nation actuel. Son objectif a été aussi de construire les idéaux d'un État-nation pacifique - sans armée -, civil et démocratique, avec une alternance électorale au pouvoir, incontestée depuis plus d'un siècle. D'autre part, teinté de danses et de musiques folkloriques et de défilés scolaires - plutôt que militaires -, le discours politique officiel transmet à la population l'idée que l'identité costaricienne est née ce jour-là.

2 Ainsi, le nationalisme contemporain, qui est devenu une idée-force des États modernes depuis le début de leur construction, au cours des processus révolutionnaires de la fin du XVIII ${ }^{e}$ siècle et tout au long du XIX ${ }^{e}$ siècles (Hobsbawm, E. $2000: 27-29$ ), a été un pilier idéologique fondamental du Costa Rica contemporain, qui a construit sa " communauté imaginaire " (Anderson, B. 1993 : 23-24) à l'image et à la ressemblance des autres nations occidentales modernes. Et, en elle-même, la fête civique - comme c'est le cas de la célébration de l'Indépendance - a également été encadrée dans le contexte de la construction de l'État, des institutions et symboles nationaux et, finalement, du nationalisme comme idéologie politique (Ihl, 0. 1996: 16-18). Cela tient-il encore aujourd'hui?

\section{Modalités commémoratives et ensemble des célébrations proposées}

3 Les célébrations du bicentenaire organisées jusqu'à présent au Costa Rica semblent avoir été principalement académiques et à peine orientées vers l'affichage public. Peut- 
être cela peut-il être attribué, comme le dit le gouvernement, à la double nécessité de confiner et d'éloigner la population en raison de la pandémie de Covid-19, d'une part, et de restreindre les dépenses publiques en situation de crise, de l'autre. Cependant, on ne peut pas s'empêcher de remarquer une certaine forme d'improvisation et de précipitation, de manque de planification, dans presque toutes les activités publiques programmées ${ }^{1}$. Un autre problème que l'on peut signaler est que les institutions publiques, en général, ont placé le Bicentenaire d'une manière ou d'une autre comme leitmotiv de leurs activités, mais celles-ci ne semblent pas avoir dépassé les pratiques usuelles: le thème est associé à toutes les activités, bien qu'elles n'aient pas été programmées dans ce but. Ainsi, il semble que la célébration ait été, en général, comme affadie. Une atmosphère générale d'apathie semble avoir régné au niveau national. Cela peut également être attribué au déclin du nationalisme du XIXe siècle, causé par la montée d'une culture globale, qui tend à éroder les cultures locales (Gruzinsky, S. 1999 : 33), ainsi que par l'affaiblissement de l'État moderne, contraint par les réformes néolibérales et globalisantes à céder une partie de sa souveraineté à des entités supraétatiques (comme les accords de libre-échange) ou à s'impliquer dans un marché mondial, qu'il ne peut pas contrôler (Zéraoui, Z. 1997-1998: 107-116).

Dans ce contexte précis, les célébrations ont été lancées le 26 février par un acte solennel aux Archives nationales, où le président de la République, Carlos Alvarado, a annoncé et dévoilé le document original de l'Acte d'Indépendance, entièrement restauré. Parallèlement, une commission gouvernementale du Bicentenaire, présidée par la ministre de la Culture, a été crée en même temps. Cependant, le président a souligné dans son discours inaugural que le programme des commémorations proposé par son gouvernement était austère, en raison des restrictions imposées par la pandémie (confinements, limitations à la mobilité...), et de la crise des finances publiques, qui a contraint les dépenses du gouvernement.

En effet, au nom du gouvernement central, seule une page Facebook intitulée «Costa Rica, 200 ans d'indépendance» expose les différentes activités programmées et communique les actions de célébration ${ }^{2}$. La date à laquelle cette page a commencé ses activités est précisément le 26 février, jour où le gouvernement a officiellement commencé les célébrations: une date bien tardive pour une célébration aussi importante. En outre, une grande partie des activités programmées portent sur des thèmes tels que la nature, la solidarité sociale, les écoles ou les jeunes. Le caractère multiethnique et multiculturel du pays est également souligné, avec un accent particulier sur les peuples autochtones, un hommage rendu à Marcus Garvey, et la célébration de l'incorporation du territoire de Nicoya au Costa Rica (1824). Cependant, ces sujets n'ont que peu ou rien à voir avec le Bicentenaire et ont été célébrés dans les mois ou les jours où leur anniversaire est habituellement célébré, de sorte que leur mention ne semble pas avoir un lien avec le sujet. Ainsi en va-t-il de l'incorporation de Nicoya, cette région ayant été saluée sur la page internet du Bicentenaire comme le berceau du folklore national, ce qui n'est en rien nouveau puisque cette pratique remonte aux années 1930 (Vargas Cullell, M. 2002 : 237-246; Hidalgo Torres, A. 2018 : 89-117). D'autre part, la page aborde également des sujets tels que les athlètes nationaux, ou les droits des femmes, généralement promus par le gouvernement. Par contre, la page mentionne des activités plus novatrices et même critiques, telles que des conférences, des séminaires, des présentations de livres, des réunions 
communautaires, des concours d'art, des forums de discussion et des concerts. Elle propose également des vidéos sur ces sujets.

6 Ainsi, cet espace semble être utilisé majoritairement pour communiquer les actions du gouvernement - comme le ferait n'importe quelle page Internet informative -, tels que son projet de déclarer le Costa Rica pays libre de l'exploration pétrolière, ou bien le plan national pour les jeunes, entre autres exemples. De cette façon, le contenu de ce site internet ressemble plus à une publicité pure et simple des projets gouvernementaux qu'aux célébrations spécifiques à une année particulière.

Et ce n'est que le 23 août que le gouvernement a annoncé le lancement de la plateforme virtuelle « 200costarica ", où l'on peut avoir accès aux informations sur les près de 400 activités entreprises depuis février. De manière significative, cette page fait référence à Facebook, où les activités menées jusqu'à présent sont enregistrées, et répète pour l'essentiel les mêmes activités qui y figurent déjà, de sorte qu'il ne semble pas être une véritable innovation.

8 Les institutions publiques décentralisées du secteur culturel ont également rejoint les festivités, mais en leur donnant une teinte plus académique quoique, parfois, avec des caractéristiques très similaires à celles du gouvernement central. Le Musée national, par exemple, réalise l'exposition intitulée «Blanco Azul y Rojo », conçue pour montrer les expressions culturelles de la fête de l'Indépendance au cours des 200 dernières années. La Bibliothèque nationale, pour sa part, a mené un travail particulièrement intense d'organisation de conférences et de tables rondes depuis 2020, plus que peutêtre toute autre institution, ce qui en fait une exception honorable. De la même manière, le Centre de recherche et de conservation du patrimoine culturel du ministère de la Culture et de la Jeunesse, dans l'édition 2020 du concours "Sauvons notre patrimoine ", a dédié son prix au Colegio Superior de Señoritas, situé à San José, qui date de beaucoup plus tard que l'époque de l'Indépendance. En plus de ce qui précède, une pièce commémorative a été émise et il y a eu des éditions spéciales de billets de loterie ainsi que de timbres-poste.

De leur côté, les municipalités ont également pris part à la célébration, mais avec des caractéristiques ressemblant fort à celles du gouvernement central. Par exemple, la municipalité de Cartago, ancienne capitale coloniale du pays et ville où fut signé l'Acte d'Indépendance en 1821, a commandé une sculpture d'une Victoire ailée à un sculpteur costaricien. Cette municipalité a lancé sur la toile une plateforme et une application qui permettent d'accéder au calendrier des célébrations. En outre, l'Asociación Cívica 29 de Octubre, apparemment liée à elle, a entrepris diverses activités commémoratives, telles que la présentation d'une salle coloniale dans le musée municipal et un défilé avec des costumes d'époque. La municipalité de San José, pour sa part, a signalé, la semaine du 23 août, qu'elle réalisera au Stade national un spectacle de drones, financé par la multinationale INTEL, auquel contribuera l'Orchestre national, une institution considérée dans le pays comme un symbole national. Selon le site Internet de cette municipalité, les activités de son plan opérationnel pour les célébrations du Bicentenaire viennent de démarrer le 20 juillet 2021, sans qu'aucune d'entre elles ne soit détaillée. Aussi, il n'y a pas de monument à inaugurer à San José : la question de la statuaire semble donc avoir été complètement abandonnée dans la capitale. Un examen des sites Internet d'autres municipalités du pays ne révèle pas de grandes célébrations prévues pour le Bicentenaire. 
10 Enfin, les universités d'État se sont également souciées de se joindre aux festivités. À l'Université du Costa Rica, l'École d'Histoire a organisé un séminaire intitulé «Le bicentenaire du Costa Rica : histoires, souvenirs, projets et futurs ", ainsi qu'une série de films (avec des productions qui n'ont rien à voir avec l'Indépendance). Dans la même université, l'École d'Études générales a organisé le "Congrès international du Bicentenaire : Défis et perspectives de l'avenir de l'Amérique centrale », et le Centre de Recherche sur l'Identité et la Culture latino-américaine (CIICLA) a programmé une série de conférences dans le cadre de l'exposition «Blanc, bleu et rouge (1821-2021)», en coopération avec le Musée national. L'Université nationale, dans la ville d'Heredia, pour sa part, a également organisé des cycles de conférences, des cours de courte durée, des présentations de livres et des programmes de télévision, ainsi que des activités du Musée de Culture populaire de l'École d'Histoire. L'Université d'État à distance (UNED), de son côté, a réalisé diverses productions audiovisuelles.

11 Bref, bien qu'il se fasse appeler le « Gouvernement du Bicentenaire », le gouvernement actuel - et l'État costaricien dans un sens plus large - ne semble pas s'être profondément engagé dans la célébration.

\section{Discours produits : les récits actuels de l'Indépendance}

Ainsi, le Bicentenaire surprend le Costa Rica, de toute évidence sans discours défini sur l'Indépendance. Sans aucun doute, le contexte qui encadre le Bicentenaire est celui de la mondialisation, du réchauffement climatique, du monde interconnecté et de la pandémie de Covid-19 (Acuña León M. et al., 2021), éléments qui contribuent tous à décourager la célébration et l'élaboration des méta-histoires de longue durée de la nation.

Les récits qui sous-tendent les activités programmées n'apparaissent pas excessivement novateurs par rapport aux traditions déjà connues. Selon le président, quatre piliers, qu'il appelle " Costa Rica historique ", "Costa Rica sain, pacifique et solidaire », " Costa Rica responsable avec l'environnement et décarboné » et " Costa Rica capable, créatif et pluriel ", donnent forme à la politique gouvernementale de célébration ${ }^{4}$. On notera qu'aucun de ces axes ne présente un discours renouvelé de quelque manière que ce soit, par rapport à ceux qui ont traditionnellement traité de la question de l'Indépendance, renvoyant plutôt à la gestion courante des politiques publiques.

D'autres propositions du gouvernement, exposées dans ses différents médias, soutiennent pleinement cette idée. L'environnement et la décarbonation, par exemple, sont indubitablement liés au changement climatique. Autre exemple, la question du pluralisme, contraire à la vision du «Costa Rica blanc » prônée par les libéraux du XIx ${ }^{\mathrm{e}}$ siècle, semble liée aux efforts du pays pour se présenter comme une destination multiculturelle, attractive pour le tourisme, image développée par l'État à partir des années 1990 (Molina Jiménez I. et S. Palmer, 2007 : 169). Enfin, le thème "Costa Rica capable, créatif et pluriel » a probablement pour objectif de remonter le moral de la population en temps de crise, et peut-être aussi de promouvoir le talent humain dans la société de l'information. Mais c'est une question récurrente dans les plans des gouvernements successifs pour attirer les investissements étrangers, en raison de la formation technologique de la population nationale. 

récemment inauguré ${ }^{5}$, d'autres axes qui accompagnent le Bicentenaire abordent les questions suivantes : le dialogue intergénérationnel, le flux de l'histoire, les soins et les droits de l'homme, la valorisation des personnes et du territoire, et la nécessité de concevoir l'avenir dans une perspective inclusive et plurielle. Cependant, il ne semble rien y avoir dans ce qui est indiqué sur cette plateforme Internet gouvernementale qui diffère des projets déjà connus.

En dehors de ce qui précède, pratiquement toutes les institutions n'ont fait que reproduire les vieux discours sur l'État et la nation, même si des tons académiques ont été entendus dans les universités publiques, ainsi que dans les rassemblements, conférences et tables rondes organisés par d'autres entités. On dirait qu'au Costa Rica, 200 ans après l'Indépendance, le récit de la patrie est épuisé.

\section{BIBLIOGRAPHIE}

Acuña León, María de los Ángeles, et. al, Guión curatorial de la exposición blanco, azul y rojo, 1821-2021, San José, Costa Rica, CIICLA, UCR, 2021.

Anderson, Benedict, Comunidades imaginadas. Reflexiones sobre el origen y la difusión del nacionalismo, México, Fondo de Cultura Económica, 1993.

Díaz Arias, David, La fiesta de Ia independencia en Costa Rica. 1821-1921, San José, Editorial UCR, 2007. Gruzinsky, Serge, La pensé métisse, Paris, Librairie Arthème Fayard, 1999.

Hidalgo Torres, Antonio J., "Las nociones de lo 'costarricense' en la música folclórica de los años 80. El caso del grupo Cantares", Káñina, Revista de Artes y Letras, Universidad de Costa Rica, XLII (1), 2018, p. 89-117.

Hobsbawm, Eric, Naciones y nacionalismos desde 1780, Barcelona, Crítica, 2000.

Ihl, Olivier, La Fête républicaine, Paris, Éditions Gallimard, 1996.

Molina Jiménez, Iván et Steven Palmer, Historia de Costa Rica, San José, EUCR, 2007.

Vargas Cullell, María Clara, De las fanfarrias a las salas de concierto: música en Costa Rica, 1840-1940, San José, EUCR, 2002.

Zéraoui, Zidane, “Globalización y problemática étnico-nacional”, Revista Estudios, San José, EUCR, n¹4-15, 1997-1998.

\section{NOTES}

1. Cela contraste fortement avec les célébrations du centenaire en 1921, qui, comme cela a été documenté, étaient planifiées un an à l'avance et comprenaient des activités telles que le dévoilement de statues, de plaques, de travaux publics, alors que tout San José fut décoré (Díaz Arias, 2007 : 211-213). Tout cela malgré le fait que ces célébrations eurent également lieu dans un 
contexte de pandémie (la grippe espagnole) et de crise économique, provoquée par la Première Guerre mondiale (Acuña León M. et al., 2021).

2. https://www.facebook.com/200CostaRica/

3. https://200costarica.go.cr

4. https://mcj.go.cr/sala-de-prensa/noticias/costa-rica-comienza-el-camino-de-laconmemoracion-de-sus-200-anos-de

5. https://200costarica.go.cr

\section{AUTEUR}

\section{EDUARDO MADRIGAL}

Dr. Eduardo Madrigal, Université du Costa RicaCostaricien, docteur en histoire de l'Université du Costa Rica et de l'Université de Toulouse II-Jean Jaurès, M. Madrigal est professeur dans les Écoles d'histoire et d'Études générales de l'Université du Costa Rica. Il a été professeur invité à l'Université de Toulouse II-Jean Jaurès de janvier à février 2015 et de janvier à février 2019. Il est, par ailleurs, chercheur affilié au Centre de Recherche sur l'Identité et la Culture LatinoAméricaines (CIICLA), de l'Université du Costa Rica.edmadm@yahoo.es 